\title{
ARTí́CULO
}

\section{Evaluación sistemática del género Helcogrammoides (Blenniformes: Tripterygiidae): Pequeños peces con grandes problemas}

Systematic evaluation of the genus Helcogrammoides (Blenniformes: Tripterygiidae): Small fishes with big problems

\section{Claudio F. Cornejo ${ }^{1 *}$, Christian M. Ibáñez ${ }^{1}$ y Cristián E. Hernández ${ }^{2}$}

${ }^{1}$ Departamento de Ecología y Biodiversidad, Facultad de Ecología y Recursos Naturales, Universidad Andres Bello, República 440, Santiago, Chile.*claudio.cornejo@uandresbello.edu

${ }^{2}$ Departamento de Zoología, Facultad de Ciencias Naturales y Oceanográficas, Universidad de Concepción, Concepción, Chile

Abstract.- In the Chilean coast, the Tripterygiidae family is represented by two species from the Helcogrammoides genus; these were originally described in the Tripterygion genus, which is currently distributed in the Mediterranean Sea and northeast Atlantic Ocean. In 1959, Rosenblatt recognizes the differences between these and the other species from Tripterygion genus and proposed the Helcogrammoides genus. A taxonomic hypothesis has not been evaluated by systematic-phylogenetic approach, which would allow to evolutionarily validate this proposal. To this date, the only comparative analysis with other species of the family, used morphological characters suggesting that the Helcogrammoides genus and the Karalepis monotypic genus, belonging to the monophyletic tribe Karalepini, which is considered to be monophyletic based on morphological synapomorphies. This study tests Rosenblatt's taxonomic hypothesis, and the monophyly of the Karalepini tribe, through a molecular and morphological phylogenetic approach. The results suggest that the Helcogrammoides genus is monophyletic, differing from Tripterygion, both morphological and molecular data, thereby, supporting Rosenblatt's hypothesis. At the same time, the molecular analysis and the concatenated molecular and morphological data, groups together the Karalepis and the Tripterygion genus in a separate clade from the Helcogrammoides genus. Thus disproving the monophyly of this tribe and suggesting that the morphological characters that supported the monophyly of Karalepini tribe could correspond to homoplasies that obscure the results of the morphological analysis based on the parsimony criterion, and explain the low a posteriori probability of this clade Bayesian analysis.

Key words: Bayesian inference, Karalepini, phylogeny, Blennioidei

Resumen.- La familia Tripterygiidae en las costas de Chile se encuentra representada por dos especies correspondientes al género Helcogrammoides; sin embargo, éstas fueron originalmente descritas en el género Tripterygion el cual se distribuye actualmente en el Mar Mediterráneo y en el noreste del océano Atlántico. En 1959, Rosenblatt reconoce las diferencias entre estas y las demás especies del género Tripterygion, proponiendo el género Helcogrammoides. En la actualidad la hipótesis taxonómica no ha sido evaluada en base a aproximaciones sistemáticas filogenéticas que permitan considerar la validez evolutiva de esta propuesta. A la fecha el único análisis comparativo realizado con otras especies de la familia utilizó caracteres morfológicos y sugiere que Helcogrammoides pertenecería a la tribu Karalepini junto al género monotípico Karalepis, tribu considerada como monofilética en base a las sinapomorfías morfológicas. En este trabajo se evalúa la hipótesis taxonómica de Rosenblatt y la monofilia de la tribu Karalepini mediante aproximaciones filogenéticas basadas en datos moleculares y morfológicos. Los resultados sugieren que el género Helcogrammoides es un género monofilético que difiere de Tripterygion tanto morfológica como molecularmente, respaldando la hipótesis de Rosenblatt. A su vez el análisis filogenético molecular, y de datos moleculares y morfológicos concatenados, agrupan el género Karalepis junto al género Tripterygion en un clado separado del género Helcogrammoides, refutando la monofilia de la tribu Karalepini y sugiriendo que los caracteres morfológicos que sustentaban la tribu podrían corresponder a homoplasias que oscurecen los resultados del análisis morfológico basado en el criterio de parsimonia y que explican la baja probabilidad a posteriori de este clado en el análisis Bayesiano.

Palabras clave: Inferencia Bayesiana, Karalepini, filogenia, Blennioidei 


\section{INTRODUCCIÓN}

La familia Tripterygiidae comprende especies de peces blenoideos asociados a hábitats rocosos de aguas heladas, temperadas, subtropicales y tropicales (Fricke 2002). Son peces óseos que presentan tres aletas dorsales, aletas pélvicas de posición ventral, y que presentan dimorfismo y dicromatismo sexual en la mayoría de las especies (Fricke 2009). En Chile, esta familia está representada por dos especies simpátricas, Helcogrammoides cunninghami (Smitt, 1898) y Helcogrammoides chilensis (Cancino, 1960). Castillo \& Pequeño (1998) señalan la distribución de $H$. chilensis entre Antofagasta (23⒊' $70^{\circ} 24^{\prime} \mathrm{W}$ ) y Talcahuano ( $36^{\circ} 45^{\prime} \mathrm{S} 73^{\circ} 08^{\prime} \mathrm{W}$ ) mientras que la distribución de H. cunninghami es señalada entre Coquimbo (2953'S $71^{\circ} 15^{\prime} \mathrm{W}$ ) y Tres Montes ( $\left.46^{\circ} 58^{\prime} \mathrm{S} 75^{\circ} 27^{\prime} \mathrm{W}\right)$. Actualmente el límite norte de la distribución de ambas especies ha sido ampliado hasta Lima (William \& Springer 2001). Adicionalmente, $H$. cunninghami ha sido reportado en las costas de Argentina entre Puerto Madryn (42 ${ }^{\circ} 49^{\prime} \mathrm{S}$

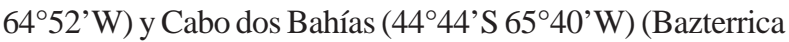
et al. 2007). Por otra parte, se encuentra una tercera especie en este género, Helcogrammoides antarcticus (Tomo 1982), que fue descrita en base a ejemplares colectados en Bahía Paraíso, territorio Antártico. Estas especies fueron originalmente descritas en el género Tripterygion Risso, 1872 (William \& Springer 2001), que actualmente se distribuye en el Mar Mediterráneo y el noreste del océano Atlántico. Al respecto, Fowler (1945) identificó a Tripterygion cunninghami Smitt, 1898 para la costa de Chile, Cancino (1955) describió a Tripterygion chilensis en una tesis no publicada, especie que fue posteriormente considerada válida por De Buen (1960) y refrendada en una diagnosis de la familia en Chile por Castillo \& Pequeño (1998). Por su parte, Rosenblatt (1959) reconoció las diferencias de estas especies de Tripterygion con las demás especies del género, proponiendo el género Helcogrammoides en base a su línea lateral continúa y compuesta solo de escamas con poros, la presencia de tentáculos nasales y supraorbitales multífidos y la ausencia de espina anal, el cual albergaría a las especies de Tripterygion de Chile. Posteriormente, tanto Gon (1990) como Castillo \& Pequeño (1998) avalan la propuesta de Rosenblatt reclasificando a las especies de Tripterygion bajo el género Helcogrammoides.

A la fecha se han realizado diversos estudios de filogenia en distintos géneros de la familia Tripterygiidae tales como Tripterygion, Helcogramma McCulloch \& Waite, 1918, Enneapterygius Rüpell, 1835, y Karalepis Hardy, 1984 (Stepien et al. 1997, Carreras-Carbonell et al. 2005, 2007; Domingues et al. 2007, Chiang \& Chen
2008, Lin 2009, Lin \& Hasting 2013). Sin embargo, estos estudios consideran una escala geográfica limitada o con un bajo número de especies (Lin 2009), razón por la que el género Helcogrammoides no ha sido incluido en ellos. Fricke (1994) realizó un estudio cladístico en base a caracteres morfológicos de la familia, identificando 8 linajes, los que actualmente son reconocidos a nivel de tribu (Fricke 2009). Dentro de estas, el género Helcogrammoides se encuentra agrupado con el género mono-específico Karalepis en la tribu Karalepini, la cual presentaría sólo dos características no exclusivas que la sustentan (Fricke 1994): la línea lateral continua y una cabeza muy reducida. Sin embargo, a la fecha las hipótesis taxonómicas de Rosenblatt y la propuesta de la tribu Karalepini no han sido evaluadas en base a aproximaciones sistemáticas filogenéticas que permitan considerar su validez. El objetivo del presente trabajo fue evaluar ambas hipótesis usando aproximaciones filogenéticas basadas en caracteres morfológicos y moleculares mediante inferencia Bayesiana y Parsimonia, para así aclarar la sistemática de este grupo de peces.

\section{Materiales y MÉTODOS}

\section{Morfología}

Con la finalidad de evaluar la asignación de las especies chilenas al género Helcogrammoides se incluyeron en este estudio a 3 especies de la tribu Tripterygiini [Enneapterygius etheostoma (Jordan \& Snyder, 1902), Tripterygion delaisi (Cardenat \& Blache, 1969), Tripterygion tartessicum Carreras-Carbonell, Pascual \& Macpherson, 2007] y a una de Notoclinini [Notoclinus compressus (Hutton, 1872)]. Con la finalidad de evaluar la consistencia monofilética de la tribu Karalepini, se incluyó a la única especie del género Karalepis (Karalepis stewarti Hardy, 1984). Además, se utilizó como grupo externo a dos especies de la familia Blennidae [Scartichthys crapulatus Williams, 1990 e Hypsoblennius sordidus (Bennett, 1828)].

La obtención de medidas morfológicas se realizó mediante la revisión tanto material depositado en la colección de peces del Museo Nacional de Historia Natural de Chile (MNHNCL), como de literatura existente (Tabla 1). Se revisó un total de 26 ejemplares correspondiente a la especie $H$. cunninghami; 30 de $H$. chilensis; 8 ejemplares de $S$. crapulatus y 5 de $H$. sordidus (Tabla 1). Además, a partir de la literatura se recopiló las medidas morfológicas para E. etheostoma, $H$. antarcticus, K. stewarti, T. delaisi, T. tartessicum y 
Tabla 1. Se muestra la fuente de la cual fueron obtenidas las medidas morfológicas. Las especies examinadas en el MNHNCL se presentan con un asterisco $\left({ }^{*}\right)$ mientras que las especies de las cuales se obtuvo las medidas morfológicas en base a la literatura se presentan con doble asterisco $\left({ }^{* *}\right)$ / Shows the source of which were obtained morphological measures. The species examined in the MNHNCL are presented with a simple mark $\left({ }^{*}\right)$, whereas the species from which the morphological measurements were obtained based on the literature are presented with double mark $(* *)$

\begin{tabular}{ll}
\hline \multicolumn{1}{c}{ Especie } & \multicolumn{1}{c}{ Fuente } \\
\hline & \\
Helcogrammoides chilensis $($ Cancino, 1960)* & MNHNCL 5559, 6872, 6873, 7415, \\
& $6874,6888,6880$ y 7421 \\
Helcogrammoides cunninghami (Smitt, 1898)* & MNHNCL 6881, 6883, 6885, 6886, \\
& 6882,6884 y 6889 \\
Helcogrammoides antarcticus (Tomo, 1982)** & Gon, 1990 \\
Enneapterygius etheostoma (Jordan \& Snyder, 1902)** & Chiang \& Chen, 2008 \\
Karalepis stewarti Hardy, 1898** & Fricke, 1994 \\
Tripterygion tartessicum Carreras-Carbonell, Pascual \& & Carreras-Carbonell, Pascual \& \\
Macpherson, 2007** & Macpherson, 2007 \\
Tripterygion delaisi Cardenat \& Blache, 1969** & Cardenat J \& Blache J, 1969 \\
Notoclinus compressus (Hutton, 1872)** & Fricke, 1994 \\
Scartichthys crapulatus Williams, 1990* & MNHNCL 6411 \\
Hypsoblennius sordidus (Bennett, 1828)* & MNHNCL 5908, 6412, 7423 \\
\hline
\end{tabular}

MNHNCL $=$ Museo Nacional de Historia Natural de Chile

N. compressus (Tabla 1). A partir de las medidas morfológicas obtenidas, tanto para los ejemplares examinados, como para los datos obtenidos desde literatura, se generó una matriz con 32 caracteres morfológicos codificados como binarios (0-1) (Tabla 2).

\section{FILOGENIA MORFOLÓGICA}

En base a la matriz binaria de 32 caracteres se reconstruyó la filogenia con inferencia Bayesiana en el programa BayesPhylogenies v 1.1 (Pagel \& Meade 2004) utilizando el modelo M2P (la tasa de ganancia y la tasa de pérdida del carácter difieren) seleccionado en base al resultado de Bayes Factor (BF) evaluado en el programa TRACER v1.5 (Rambaut et al. 2014), corriendo 4 cadenas de Markov Monte Carlo (MCMC), realizando 5.000.000 de generaciones y quemando el $10 \%$ de los árboles. Además, se reconstruyó la filogenia en base al criterio de Máxima Parsimonia (MP) (Parsimonia de Wagner, B\&B) en el programa PAUP V.4.0 (Swofford 2002) utilizando 100.000 réplicas de Bootstrap. Para reconocer las sinapomorfías morfológicas de los grupos de interés (género Helcogrammoides y tribu Karalepini) se realizó el mapeo de sinapomorfías en el programa TNT v1.5 (Goloboff \& Catalano 2016).
EXTRACCIÓN DE ADN, SECUENCIACIÓN Y AMPLIFICACIÓN A partir de 3 ejemplares de $H$. chilensis, 2 de $H$. cunninghami, uno de $H$. sordidus y uno de $S$. crapulatus recolectados entre Las Cruces (3330'11,32'"S;

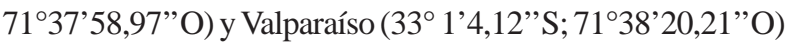
en la costa central de Chile, se extrajo ADN genómico desde tejido muscular usando el procedimiento estándar fenol:cloroformo y precipitación con etanol-isomil (Sambrook et al. 1989), previa digestión de éstos en una solución compuesta por $800 \mu \mathrm{L}$ de buffer lisis TE (10nm tris pH 7,5- 1 mM EDTA), $100 \mu \mathrm{L}$ de detergente Twin 20, y $10 \mu \mathrm{L}$ de Proteinasa K $(20 \mathrm{mg} / \mathrm{mL})$ durante $2 \mathrm{~h}$ a $25^{\circ} \mathrm{C}$. Una muestra del extracto fue sembrada en gel de agarosa al $1 \%$ para corroborar la cantidad y calidad relativa del ADN. Luego se amplificó el gen 12S por medio de la reacción en cadena de la polimerasa (PCR: Palumbi 1996). Para esto se diseñaron partidores adecuados a los especímenes analizados. Los partidores generados (LBE-06 H, TTTACACATGCA AGTCTCCGCA y LBE-07 L, CACCTTCCGGTACACT TACCATG), fueron utilizados en una concentración de $10 \mathrm{pmol} / \mu \mathrm{L}$. La amplificación mediante PCR fue realizada en $25 \mu \mathrm{L}$ de volumen total, que contenía $2 \mu \mathrm{L}$ de $\mathrm{ADN}$ genómico de $5 \mathrm{ng} / \mu \mathrm{L}, 12 \mu \mathrm{L}$ de agua bidestilada (dd $\mathrm{H}_{2} \mathrm{O}$ ), $3 \mu \mathrm{L}$ de Buffer PCR 10x, $3 \mu \mathrm{L}$ de cloruro de magnesio de $50 \mathrm{mM}\left(\mathrm{MgCl}_{2}\right), 3 \mu \mathrm{L}$ de 
Tabla 2. Codificación de los caracteres morfológicos como datos binarios para los análisis filogenéticos. LE= Longitud estándar / Morphological characters codification as binary data for phylogenetic analysis. LE= Standard length

\begin{tabular}{|c|c|c|c|}
\hline $\begin{array}{l}\text { Número } \\
\text { de } \\
\text { carácter }\end{array}$ & Carácter & 0 & 1 \\
\hline 1 & Longitud cefálica & Inferior al $25 \%$ de la LE & Superior al $25 \%$ de la LE \\
\hline 2 & Longitud preanal & Hasta el $50 \%$ de la LE & Superior al $50 \%$ de la LE \\
\hline 3 & Longitud predorsal & Inferior al $20 \%$ de la LE & Superior al $20 \%$ de la LE \\
\hline 4 & Altura máxima del cuerpo & Menor al $22 \%$ de la LE & Superior al $22 \%$ de la LE \\
\hline 5 & Altura del pedúnculo caudal & Menor al $8 \%$ de la LE & Superior al $8 \%$ de la LE \\
\hline 6 & Diámetro ocular & Menor al $8 \%$ de la LE & Superior al $8 \%$ de la LE \\
\hline 7 & Distancia interocular & Menor al $5 \%$ de la LE & Superior al $5 \%$ de la LE \\
\hline 8 & Base de las espinas dorsales & Menor al $40 \%$ de la LE & Superior al $40 \%$ de la LE \\
\hline 9 & Base de los radios dorsales & Menor al $30 \%$ de la LE & Superior al $30 \%$ de la LE \\
\hline 10 & Número de aletas dorsales & 1 Aleta dorsal & 3 Aletas dorsales \\
\hline 11 & Largo de la aleta pectoral & Inferior al $30 \%$ de la LE & Mayor al $30 \%$ de la LE \\
\hline 12 & Largo de la aleta pélvica & Inferior al $15 \%$ de la LE & Mayor al $15 \%$ de la LE \\
\hline 13 & Base de la aleta anal & Inferior al $45 \%$ de la LE & Mayor al $45 \%$ de la LE \\
\hline 14 & Número de espinas dorsales & Hasta 15 & Más de 15 \\
\hline 15 & Número de radios dorsales & Hasta 15 & Más de 15 \\
\hline 16 & Espina en la aleta anal & Ausente & Presente \\
\hline 17 & Radios en la aleta anal & Hasta 20 & Más de 20 \\
\hline 18 & Forma de la aleta caudal & Redonda & Truncada \\
\hline 19 & Espinas en la aleta pélvica & Ausente & Presente \\
\hline 20 & Radios en la aleta pélvica & 2 Radios pélvicos & 4 Radios pélvicos \\
\hline 21 & Escamas en el cuerpo & Ausentes & Presentes \\
\hline 22 & Radios de la aleta pectoral & Solo radios simples & Radios simples y ramificados \\
\hline 23 & Forma de la aleta pectoral & Redondeada & Cincelada / Lanceolada \\
\hline 24 & Continuidad de la línea lateral & Discontinua & Continua \\
\hline 25 & Forma de la línea lateral & Descenso abrupta & Descenso suave \\
\hline 26 & Composición de la línea lateral & Poros sensoriales & Poros y túbulos sensoriales \\
\hline 27 & Forma de la cabeza & Triangular & Redondeada \\
\hline 28 & Posición de la boca & Terminal & Subterminal \\
\hline 29 & Cirros nucales & Ausentes & Presentes \\
\hline 30 & Forma de los cirros nasales & Simples & Ramificados \\
\hline 31 & Número de cirros supraoculares & Solo 1 cirro & Varios cirros \\
\hline 32 & Longitud máxima & Inferior a $\operatorname{los} 10 \mathrm{~cm}$ & Superior a los $10 \mathrm{~cm}$ \\
\hline
\end{tabular}


desoxinucleotido de trifosfato de 2,5 nM (dNTP)m 0,5 $\mu \mathrm{L}$ de primer $\mathrm{L}, 0,5 \mu \mathrm{L}$ de primer $\mathrm{H}, \mathrm{y} 1 \mu \mathrm{L}$ de Taq DNA polimerasa de $5 \mathrm{u} / \mu \mathrm{L}$ (BIOTOOLS, España). La amplificación por PCR fueron realizadas con el siguiente protocolo de termociclado, en 35 ciclos: Denaturación inicial a $94^{\circ} \mathrm{C}$ durante $5 \mathrm{~min}$, denaturación a $95^{\circ} \mathrm{C}$ por $45 \mathrm{~s}$, alineamiento a $61^{\circ} \mathrm{C}$ por $55 \mathrm{~s}$, extensión a $72^{\circ} \mathrm{C}$ por $90 \mathrm{~s}$ y extensión final a $72^{\circ} \mathrm{C}$ durante $5 \mathrm{~min}$. El tamaño del producto PCR fue chequeado en un gel de agarosa al $1,5 \%$. Finalmente, el ADN amplificado fue purificado con el kit QIAquick PCR de acuerdo al protocolo del fabricante (Qiagen, USA). La secuenciación se realizó en ambas direcciones (Foward y Reverse) en un secuenciador automático de ADN (Model ABI3100, Applied Biosystems) bajo las siguientes condiciones: Polímero POP6, Byg Dye Terminator V.3, voltaje de $12 \mathrm{~V}$, temperatura de $50^{\circ} \mathrm{C}$ y un tiempo de corrido de $7.500 \mathrm{~s}$.

\section{Filogenia MOLECULAR}

Para el análisis molecular se utilizó la secuencia del gen mitocondrial 12S rRNA obtenidas en este estudio en conjunto con secuencias de GenBank (Tabla 3). La reconstrucción filogenética se realizó en el programa BayesPhylogenies V.1.1 (Pagel \& Meade 2004) mediante inferencia Bayesiana utilizando el modelo de sustitución Kimura 2 parámetros (K2P, Kimura 1980), corriendo 4 cadenas MCMC, 5.000.000 de generaciones y quemando el 10\% de los árboles guardados. El modelo K2P fue seleccionado en base a su valor de AIC evaluado en el programa MEGA V 7.0 (Kumar et al. 2016). Además, se reconstruyó la filogenia en base al criterio de MP (Parsimonia de Wagner) en el programa PAUP V.4.0 (Swofford 2003) utilizando 100.000 réplicas de Bootstrap.

\section{ANÁLISIS de DATOS CONCATENADOS}

Para este análisis, se realizó una matriz particionada de los datos morfológicos y las secuencias del gen mitocondrial 12S rRNA, generando una matriz con una partición para cada tipo de dato, y siendo cada una de estas trabajada en base a los parámetros previamente detallados. Se realizó esta reconstrucción filogenética en base al método Bayesiano en el programa BayesPhylogenies V.1.1 (Pagel \& Meade 2004) corriendo 4 cadenas MCMC, con 5.000.000 de generaciones y quemando el 10\% de los árboles guardados.

\section{Resultados}

El análisis filogenético morfológico en base a la MP reconoció un total de 30 caracteres filogenéticamente informativos. Los análisis filogenéticos morfológicos, tanto MP como Bayesiano, mostraron similar topología. Ambos muestran que el clado correspondiente a la familia Tripterygiidae presenta altos valores de sustento de nodo y probabilidad a posteriori (Fig. 1: $\mathrm{MP}=100 \%$; $\mathrm{PP}=1,0)$. A su vez la familia Tripterygiidae se subdivide en 2 clados: El primero de los clados agrupa a especies de la subfamilia Tripterygiinae (Tripterygion tartessicum, Enneapterygius etheostoma, Tripterygion delaisi) con Notoclinus compressus (subfamilia Notoclininae) como especie hermana. Sin embargo, tanto en este clado, como los nodos internos tienen bajos sustento ( $<50 \%$ en MP) y probabilidad a posteriori $(<$ 0,3 PP). El segundo clado agrupa a los géneros Karalepis y Helcogrammoides en la denominada tribu Karalepini, el cual muestra valores bajos de sustento (67\% en MP) y probabilidad a posteriori (0,47 PP). El mapeo de sinapomorfías revela que los caracteres

Tabla 3. Fuente de las Secuencias del gen 12S rRNA utilizadas en los análisis filogenéticos / Source of the $12 \mathrm{~S}$ rRNA gene sequences used on phylogenetic analysis

\begin{tabular}{ll}
\hline \multicolumn{1}{c}{ Especie } & \multicolumn{1}{c}{ Código de Acceso (GenBank) } \\
\hline Helcogrammoides chilensis (Cancino, 1960) & MF458192; MF458193; MF458194 \\
Helcogrammoides cunninghami (Smitt, 1898) & MF458195; MF458196 \\
Karalepis stewarti (Hardy, 1984) & U90383.1 \\
Enneapterygius etheostoma (Jordan \& Snyder, 1902) & U90385.1 \\
Tripterygion delaisi (Cardenat \& Blache, 1969) & AJ872171.1; AJ872161.1 \\
Notoclinus compressus (Hutton, 1872) & U90386.1 \\
Hypsoblennius sordidus (Bennett, 1828) & MF458197 \\
Scartichthys crapulatus Williams, 1990 & MF458198 \\
\hline
\end{tabular}




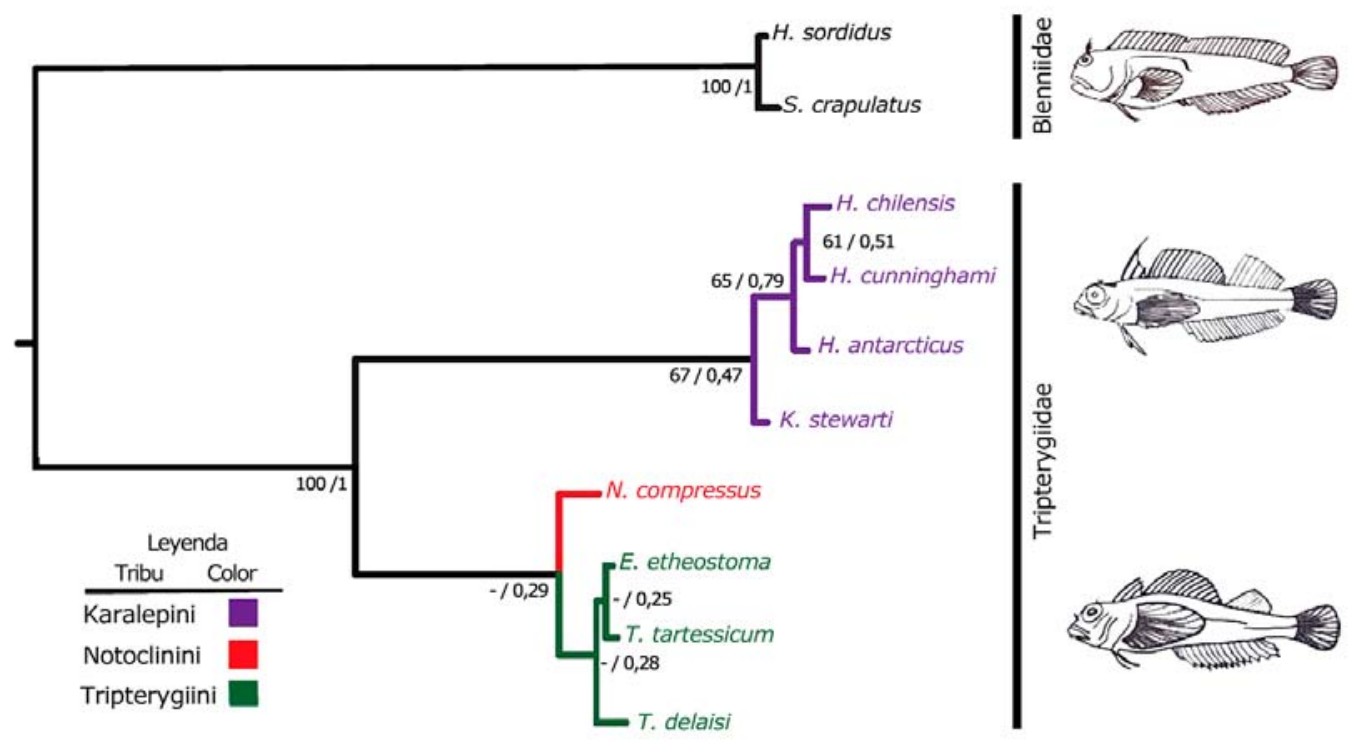

Figura 1. Reconstrucción filogenética basada en caracteres morfológicos bajo inferencia Bayesiana. El primer número antes de cada nodo representa el valor de sustento de Bootstrap en MP y el segundo valor la probabilidad posterior. Los colores de las ramas del árbol señalan las tribus a las cuales pertenecen las especies analizadas (ver leyenda) / Phylogenetic reconstruction based on morphological characters under Bayesian inference. The first number before each node represents the Bootstrap support value of MP and the second the posterior probability value. The colors of the tree branches indicate the tribes to which belong the species analyzed (see legend)

sinapomórficos del clado conformado por el género Helcogrammoides corresponden al largo de la aleta pectoral, ausencia de espina en la aleta anal, ausencia de espina en la aleta pélvica, línea lateral compuesta solo de poros sensoriales y cirros nasales ramificados (Tabla 4). Mientras que las sinapomorfías que sustentan a la tribu Karalepini corresponden a la longitud cefálica, la altura del pedúnculo caudal, el diámetro ocular, presentar una línea lateral continua y con un descenso suave desde la cabeza al pedúnculo caudal (Tabla 4).

El análisis filogenético molecular en base a la secuencia del gen 12S rRNA, mediante la MP reconoció un total de 78 sitios filogenéticamente informativos. Los análisis filogenéticos moleculares inferidos mediante inferencia Bayesiana y máxima parsimonia presentaron similar topología, al igual que en el análisis filogenético morfológico, soportan el clado de la familia Tripterygiidae $(100 \%$ MP y PP=1,0) (Fig. 2). Si bien en este análisis la familia también se agrupa en 2 clados, a diferencia de los resultados morfológicos, en este caso el género Helcogrammoides resulta ser un clado independiente con alto sustento (98\% MP) y probabilidad a posteriori $(\mathrm{PP}=1,0)$ que no incluye a la especie Karalepis stewarti (Fig. 2). De esta forma la tribu Karalepini no sería un grupo natural y constituiría un clado polifilético. Dentro del clado Helcogrammoides, los individuos secuenciados se adscriben a las especies respectivas formando grupos monofiléticos con fuerte sustento (> 98\% MP) y probabilidad a posteriori ( $\mathrm{PP}=1,0)$. Por otra parte, el clado formado por el resto de las especies de la familia se subdivide en 2 clados $(\mathrm{PP}=0,79)$ : uno que alberga sólo a $N$. compressus, representante de la subfamilia

Tabla 4. Lista de sinapomorfías para los grupos de interés. LE= Longitud estándar / List of synapomorphies for interest groups. $\mathrm{LE}=$ Standard length

\begin{tabular}{ll}
\hline \multicolumn{1}{c}{ Grupo de interés } & \multicolumn{1}{c}{ Sinapomorfias (Número de carácter) } \\
\hline Género Helcogrammoides & $\begin{array}{l}\text { Aleta pectoral mayor al } 30 \% \text { de la LE, } \\
\text { Ausencia de espina anal, Ausencia de } \\
\text { espinas en aletas pélvicas, línea lateral } \\
\text { compuesta solo por poros sensoriales y } \\
\text { presentan cirros nasales ramificados }\end{array}$ \\
Tribu Karalepini & $\begin{array}{l}\text { Longitud cefálica inferior al } 25 \% \text { de la LE, } \\
\text { 8\% del pedúnculo caudal inferior al }\end{array}$ \\
un descenso suave desde la cabeza al \\
pedúnculo caudal y línea lateral compuesta \\
solo por poros sensoriales
\end{tabular}




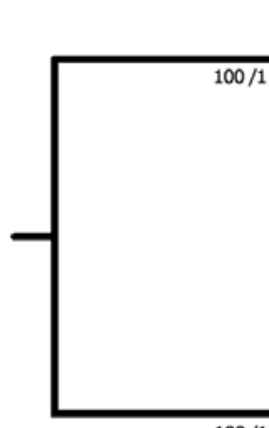

S. crapulatus
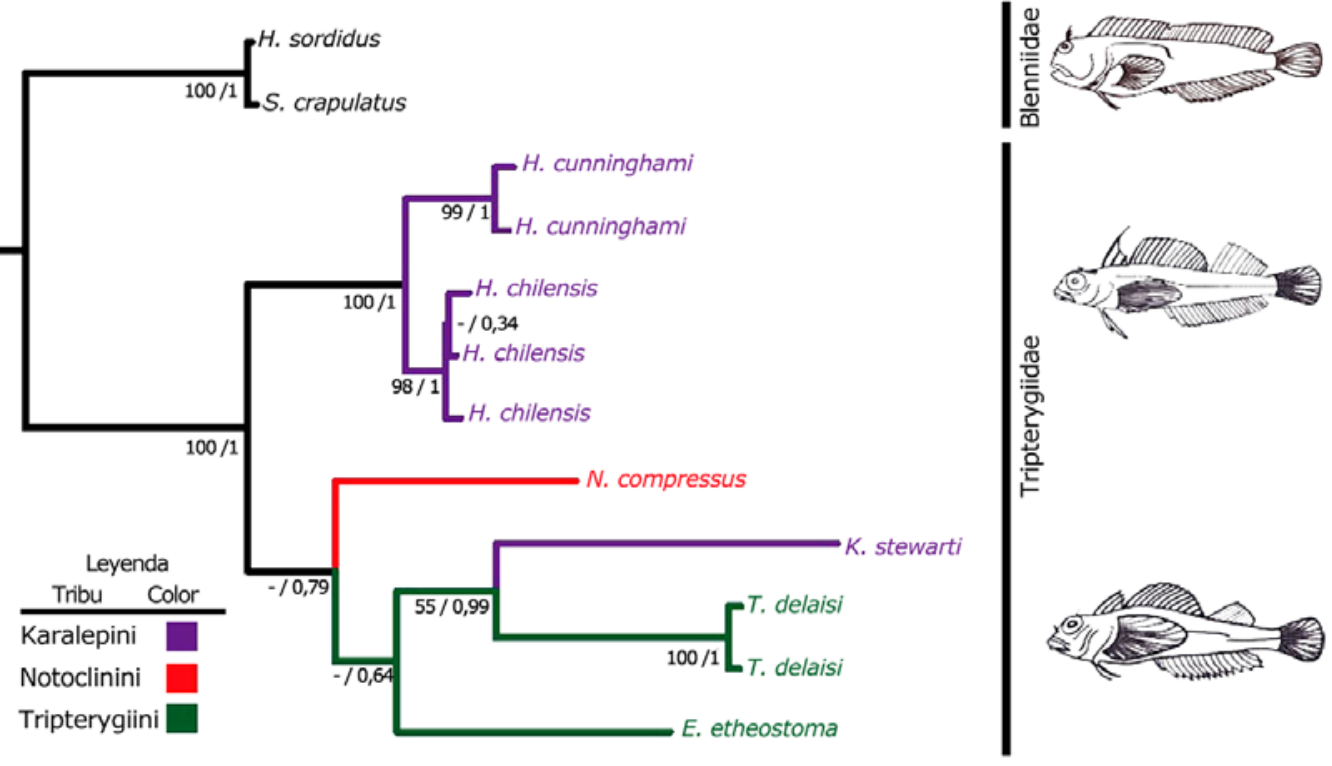

Figura 2. Reconstrucción filogenética basada en caracteres moleculares (12S rRNA) bajo inferencia Bayesiana. El primer número antes de cada nodo representa el valor de sustento de Bootstrap en MP y el segundo valor la probabilidad posterior. Los colores de las ramas del árbol señalan las tribus a las cuales pertenecen las especies analizadas (ver leyenda) / Phylogenetic reconstruction based on molecular characters (12S rRNA) under Bayesian inference. The first number before each node represent the Bootstrap support value of MP and the second the posterior probability value. The colors of the tree branches indicate the tribes to which belong the species analyzed (see legend)

Notoclininae; y el otro clado con un 0,64 de PP incorpora las especies de la tribu Tripterygiini, dentro de este clado la especie K. stewarti se presenta como especie hermana de las especies del género Tripterygion (55\% MP y 0,99 $\mathrm{PP})$.

La reconstrucción filogenética basada en datos morfológicos y genéticos concatenados (Fig. 3), reafirma los resultados de los análisis basados exclusivamente en datos moleculares. Sin embargo, en este caso la probabilidad a posteriori en cada nodo es siempre mayor o igual a 0,9 , destacando el clado compuesto por las especies del género Helcogrammoides, que presentan sólo probabilidades a posteriori de 1,0.

\section{Discusión}

Originalmente las especies del género Helcogrammoides fueron clasificadas en el género Tripterygion (Williams \& Springer 2001), sin embargo, Rosenblatt reconoció las diferencias de estas con el resto de las especies del género, proponiendo el género Helcogrammoides. Gon (1990) y Castillo \& Pequeño (1998) validaron y reclasificaron las especies en el actual género. Sin embargo, Helcogrammoides no fue objeto de estudio de la sistemática filogenética sino hasta el trabajo filogenético morfológico de Fricke (1994), que sugiere la tribu Karalepini para incluir tanto a Karalepis stewarti como a las especies del género Helcogrammoides, pero sustentada sólo en dos caracteres lábiles y no exclusivos a esta tribu (i.e., la línea lateral continua y una cabeza muy reducida).

Este estudio constituye el primer análisis sistemático filogenético molecular que intenta dilucidar las asignaciones taxonómicas propuestas para el género Helcogrammoides, y los resultados sustentan que el género Helcogrammoides es monofilético, difiriendo de Tripterygion tanto morfológica como molecularmente, respaldando la hipótesis original de Rosenblatt. A su vez el análisis filogenético molecular, y de datos moleculares y morfológicos concatenados, agrupa el género Karalepis junto al género Tripterygion en un clado separado del género Helcogrammoides, refutando la monofilia de la tribu Karalepini y sugiriendo que los caracteres morfológicos que sustentaban la tribu podrían corresponder a homoplasias que oscurecen los resultados del análisis filogenético morfológico basados en el criterio de parsimonia y que explican la baja probabilidad $a$ posteriori de este clado en el análisis Bayesiano. 

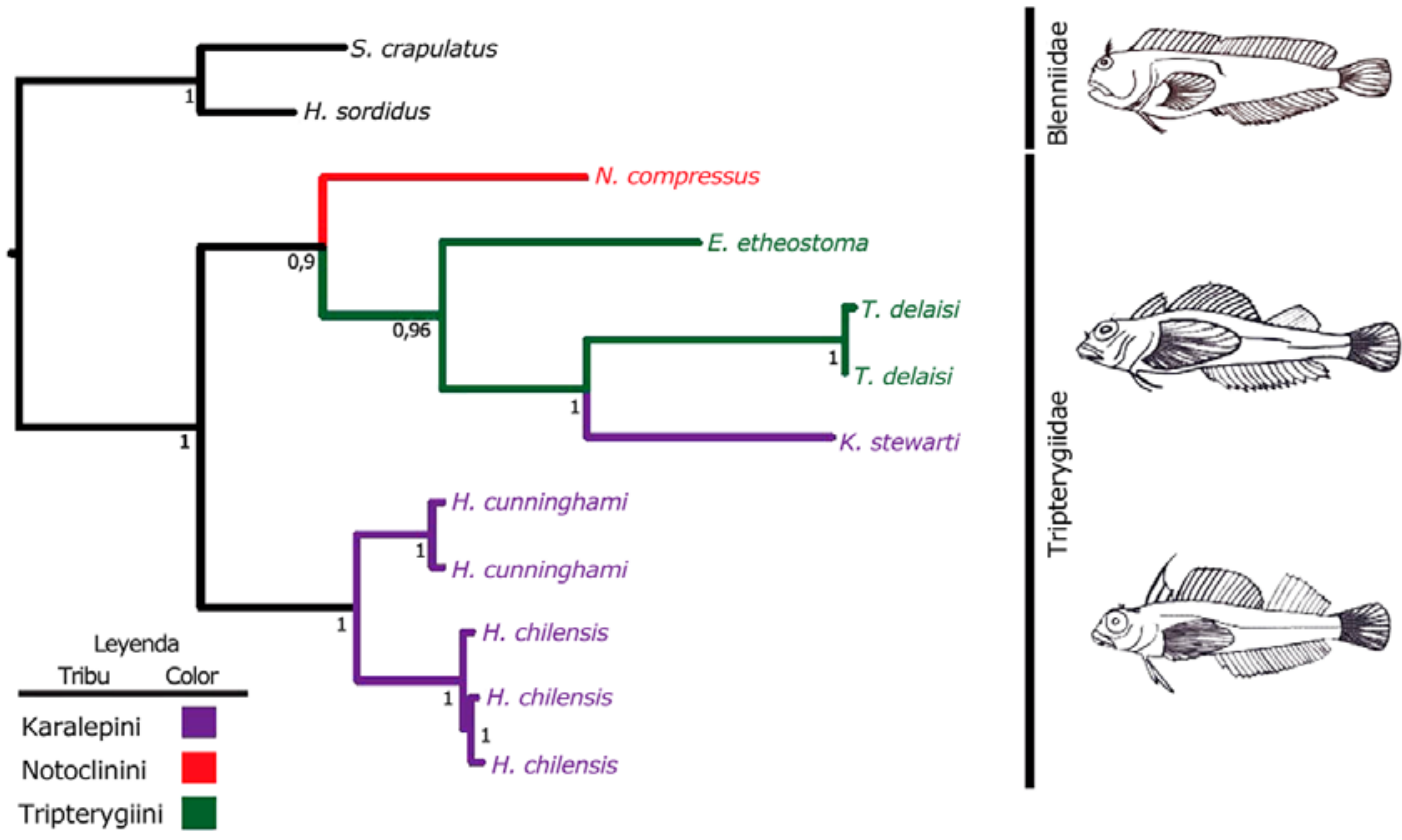

Figura 3. Reconstrucción filogenética basada en los datos morfológicos y moleculares (12S rRNA) concatenados bajo inferencia Bayesiana. El número antes de cada nodo representa el valor de probabilidad posterior. Los colores de las ramas del árbol señalan las tribus a las cuales pertenecen las especies analizadas (ver leyenda) / Phylogenetic reconstruction based on morphological and molecular characters (12S rRNA) concatenated under Bayesian inference. The first number before each node represent the Bootstrap support value of MP and the second the posterior probability value. The colors of the tree branches indicate the tribes to which belong the species analyzed (see legend)

Un avance previo a la clarificación de la taxonomía de Helcogrammoides lo logró Castillo \& Pequeño (1998), quienes por primera vez sistematizan de modo muy definido las diferencias morfológicas entre las dos especies simpátricas de Helcogrammoides que habitan la costa de Chile, resaltando dentro de las medidas morfometrícas la longitud pre anal, la altura máxima del cuerpo, la longitud de la aleta pélvica y la altura mínima del pedúnculo caudal. La revisión morfológica de los ejemplares del MNHNCL muestra congruencia en la longitud de la aleta pélvica como una medida que da cuenta de la diferenciación de estas especies. Castillo \& Pequeño (1998) señalan como principal diferencia entre ambas especies la espina prolongada en la primera aleta dorsal $\left(1^{\circ}\right.$ espina para $H$. cunninghami, $2^{\circ}$ espina para $H$. chilensis). William \& Springer (2001) señalan que el caso de $H$. antarcticus es similar al de $H$. cunninghami $\left(1^{\circ}\right.$ espina de la primera aleta dorsal). Sin embargo, basándose en el trabajo de Gon (1990), estos autores señalan que mientras $H$. cunninghami y $H$. chilensis presentan el vientre carente de escamas, $H$. antarcticus presenta escamas cicloideas en su vientre, lo que constituiría una autopomorfía de la especie y por tanto un carácter diagnóstico para diferenciar ambas especies que presentan en común una prolongada primera espina de la primera aleta dorsal. Sin embargo, sobre la base de: la mala preservación del material tipo de H. antarcticus (ver Gon 1990), la ausencia de blenoídeos en Antártica (Williams \& Springer 2001), y el hecho que no se ha podido recolectar nuevamente de la localidad tipo (Hüne M, com. pers.) ${ }^{1}$, permite sugerir a esta especie como nomen dubium (Art. 75 CINZ).

Este trabajo brinda nueva evidencia que sustenta la hipótesis de Rosenblatt (1959), mediante el uso de técnicas filogenéticas en base tanto a caracteres morfológicos como genéticos (Figs. 1-3). Los resultados demuestran que las especies pertenecientes al género Helcogrammoides se encuentran debidamente diferenciadas de las especies del género Tripterygion, a su vez que Helcogrammoides es un grupo monofilético sustentado tanto en análisis morfológicos como genéticos (Morfológico, 65\% en MP y 0,79 de PP; Genético, 100\% en MP y 1,0 de PP; Concatenado, 1,0 de $\mathrm{PP})$, coincidiendo parte de los caracteres indicados por Rosenblatt (1959) como diagnóstico con los resultados del mapeo de sinapomorfías. Por otra parte, estos

${ }^{1}$ Mathias Hüne, 2015. Fundación Ictiológica. <mathiashune@gmail.com> 
resultados no sustentan la hipótesis de Fricke (1994) de la existencia de la Tribu Karalepini, dado que tanto caracteres genéticos y datos concatenados muestran fuerte evidencia de que $K$. stewarti no forma parte del clado de los Helcogrammoides (Figs. 2 y 3). Si bien, los datos morfológicos muestran la existencia de la Tribu Karalepini (Fig. 1), su probabilidad a posteriori es muy baja $(0,47)$, poniendo en cuestionamiento el carácter monofilético de esta tribu sólo basada en caracteres morfológicos no exclusivos y lábiles. Por lo cual, los datos morfológicos podrían estar sujetos a fuertes procesos de convergencia o paralelismos que oscurecen las relaciones filogenéticas, aunque estos caracteres sigan siendo útiles para la clasificación taxonómica. Los resultados de los análisis en base a caracteres genéticos y datos concatenados agrupan al género Karalepis con las especies del género Tripterygion presentando un valor de sustento de nodo y probabilidad a posteriori que indica una alta consistencia de este clado (Genético: 0,94 de PP; Concatenado: 1,0 de PP). Sin embargo, estos resultados deben ser tomados con cautela tanto por el bajo número de especies utilizadas (ver Lin \& Hasting 2013), como por el uso de solo un marcador molecular. De hecho, la familia Tripterygiidae es una familia que comprende 163 especies albergadas en 31 géneros (Fricke 2009), en este estudio solo fueron incluidas 8 especies de esta familia, representantes de solo 4 géneros. El número de especies incluidas en este estudio es producto de la compatibilidad entre los objetivos de este estudio y el estado del arte para esta familia. La evaluación de la tribu Karalepini requiere la inclusión de K. stewarti en el análisis, especie de la cual solo está disponible la secuencia del gen 12S rRNA, siendo por tanto este el primer filtro utilizado en la selección de especies. A su vez, la precaria descripción morfológica de algunas especies de la familia que presentan este gen o la incompatibilidad de la descripción con las medidas morfológicas utilizadas en este estudio actuó como segundo filtro para seleccionar el número de especies, por tanto los resultados estarían sujetos a la influencia del bajo número de especies, lo que puede cambiar los resultados obtenidos mediante la adición de nuevas especies o distintos marcadores moleculares al estudio. Debido a que el gen $12 \mathrm{~S}$ es muy conservado en peces, solo se secuenció un bajo número de ejemplares (1-3 individuos) de cada una. No obstante, la consistencia de los resultados actuales, invita a revisar exhaustivamente las relaciones filogenéticas de la familia, resaltando la importancia de los caracteres morfológicos para la clasificación taxonómica cuando estos caracteres no estén sujetos a homoplasias, y a enfatizar el uso de caracteres moleculares para la caracterización sistemática filogenética dado que nos entrega evidencia robusta de la historia evolutiva de la diversa familia Tripterygiidae.

\section{Agradecimientos}

Queremos agradecer al Sr. Augusto Cornejo por facilitar el acceso a los ejemplares examinados en el Museo Nacional de Historia Natural, Santiago, Chile. También queremos dar las gracias a Mathias Hüne (Fundación Ictiológica) por proveer literatura clásica muy atingente al trabajo. La investigación de CEH fue apoyada por los proyectos FONDECYT 1140692 - 1170815, y la Dirección de Relaciones Internacionales, Universidad de Concepción, con el programa de colaboración internacional entre la St. Cloud State University y la Universidad de Concepción.

\section{LITERATURA CITADA}

Bazterrica M, B Silliman, J Hidalgo, C Crain \& M Bertness. 2007. Limpet grazing on a physically stressful Patagonian rocky shore. Journal of Experimental Marine Biology and Ecology 353: 22-34.

Cancino C. 1955. Peces de las pozas litorales de Chile Central. Memoria de Profesor de Biología y Química, Facultad de Filosofía y Educación, Universidad de Chile, Santiago, 135 pp.

Cancino C. 1960. Tripterygion chilensis. En: De Buen F (1960). Los peje-sapos (Familia Gobiesocidae) en Chile. Revista de Biología Marina 10(1-3): 69-82.

Cardenat J \& J Blache. 1969. Description d'une espèce nouvelle Tripterygion delaisi sp. nov., provenant de l'île de Gorée (Sénégal) (Pisces, Clinidae), Bulletin du Muséum National d'Histoire Naturelle; 1ère Série; Tome 41(5): 10971105.

Carreras-Carbonell J, E Macpherson \& M Pascual. 2005. Rapid radiation and cryptic speciation in Mediterranean triplefin blennies (Pisces: Tripterygiidae) combining multiples genes. Molecular Phylogenetics and Evolution 37(3): 751-761.

Carreras-Carbonell J, M Pascual \& E Macpherson. 2007. A review of the Tripterygion tripnopterus (Risso, 1810) complex, with description of a new species from Mediterranean sea (Teleostei: Tripterygiidae). Scientia Marina 7(1): 75-86.

Castillo R \& G Pequeño. 1998. Sinopsis de Tripterygiidae de Chile (Osteichthyes: Perciformes). Gayana Zoología 62(2): 109-133.

Chiang M \& I Chen. 2008. Taxonomic review and molecular phylogeny of the triplefin genus Enneapterygius (Teleostei: Tripterygiidae) from Taiwan, with descriptions of two new sepecies. The Raffles Bulletin of Zoology, Supplement 19: 183-201. 
De Buen F. 1960. Los peje-sapos (Familia Gobiesocidae) en Chile. Revista de Biología Marina 10(1-3): 69-82.

Domingues V, V Almada, R Santos, A Brito \& G Bernardi. 2007. Phylogeography and evolution of the triplefin Tripterygion delaisi (Pisces, Blennioidei). Marine Biology 150(3): 509-519.

Fricke R. 1994. Tripterygiid Fishes of Australia, New Zealand and the Southwest Pacific Ocean (Teleostei), 585 pp. Koeltz Scientific Books, Koenigstein.

Fricke R. 2002. Tripterygiid fishes of New Caledonia, with zoogeographical remarks. Environmental Biology of Fishes 65: $175-198$.

Fricke R. 2009. Systematics of the Tripterygiidae (Triplefins). In: Patzner RA, EJ Gonçalves, PA Hastings \& BG Kapoor (eds). The biology of blennies, pp. 31-67. Science Publishers, Enfield.

Goloboff P \& S Catalano. 2016. TNT version 1.5, including a full implementation of phylogenetics morphometrics. Cladistics 32(3): 221-238.

Gon O. 1990. Tripterygiidae. In: Gon O \& PC Heemstra (eds). Fishes of the Southern Ocean, pp. 400-401. J.L.B. Smith Institute of Ichthyology, Grahamstown.

Kimura M. 1980. A simple method for estimating evolutionary rate of base substitutions through comparative studies of nucleotide sequences. Journal of Molecular Evolution 16: 111-120.

Kumar S, G Stecher \& K Tamura. 2016. MEGA7: Molecular Evolutionary Genetics Analysis V 7.0. Molecular Biology and Evolution 33: 1870-1874.

Lin H-C. 2009. Evolution of the suborder Blennioidei: Phylogeny and phylogeography of a shallow water fish clade. Thesis PhD, University of California, San Diego, 150 pp. <http://escholarship.org/uc/item/82c1r9xr>
Lin H-C \& P Hastings. 2013. Phylogeny and biogeography of a shallow water fish clade (Teleostei: Blenniiformes). BMC Evolutionary Biology 13: 210.

Pagel M \& A Meade. 2004. A phylogenetic mixture model for detecting pattern heterogeneity in gene sequence or character-state data. Systematic Biology 53: 571-581.

Rambaut A, M Suchard, D Xie \& A Drummond. 2014. Tracer v1.5, <http://beast.bio.ed.ac.uk/Tracer>

Rosenblatt R. 1959. A revisionary study of the Blennioid fish family Tripterygiidae. Ph. D. Thesis, University of California, Los Angeles, 376 pp.

Sambrook J, E Fritschi \& T Maniatis. 1989. Molecular cloning: A laboratory manual, 1626 pp. Cold Spring Harbor Laboratory Press, New York.

Stepien C, A Dillon, D Brooks, K Chase \& A Hubers. 1997. The evolution of Blennioids fishes based on an analysis of mitochondrial 12S rDna. In: Kocher T \& C Stepien (eds). Molecular systematics of fishes, pp. 245-270. Academic Press, San Diego.

Swofford D. 2002. PAUP*. Phylogenetic Analysis Using Parsimony (*and Other Methods). v. 4.0. Sinauer Associates, Sunderland.

Tomo A. 1982. Contribución al conocimiento de la fauna ictiológica del sector Antártico Argentino. Dirección Nacional del Antártico, Instituto Antártico Argentino, Publicación 14: 1-242.

Williams J \& V Springer. 2001. Review of the South American-Antarctic Triplefin fish genus Helcogrammoides (Perciformes: Tripterygiidae). International Journal of Tropical Biology and Conservation 49: 117-123.

Recibido el 29 de noviembre de 2016 y aceptado el 13 de junio de 2017

Editor: Claudia Bustos D. 